This full text version, available on TeesRep, is the PDF (final version) of:

Henaghan, J. L. et al. (2008) 'The effect of structured exercise classes and a lifestyle intervention on cardiovascular risk factors in primary schoolchildren: An exploratory trial (the A-CLASS Project)', Pediatric Exercise Science, 20 (2), pp.169-180.

For details regarding the final published version please click on the following link:

http://hk.humankinetics.com/eJournalMedia/pdfs/15797.pdf

When citing this source, please use the final published version as above.

This document was downloaded from http://tees.openrepository.com/tees/handle/10149/93428 Please do not use this version for citation purposes.

All items in TeesRep are protected by copyright, with all rights reserved, unless otherwise indicated. 


\title{
The Effect of Structured Exercise Classes and a Lifestyle Intervention on Cardiovascular Risk Factors in Primary Schoolchildren: An Exploratory* Trial (The A-CLASS Project)
}

\section{Jayne Henaghan, Nicola McWhannell, Lawrence Foweather, N. Tim Cable, Alan M. Batterham, Gareth Stratton, and Keith P. George}

\begin{abstract}
This exploratory trial evaluates the effect of a structured exercise (STEX) or lifestyle intervention (PASS) program upon cardiovascular (CV) disease risk factors in children. Sixty-one schoolchildren were randomly assigned by school to an intervention or control (CON) condition. The effect of the STEX (compared with $\mathrm{CON}$ ) was a mean benefit of $-0.018 \mathrm{~mm}$ for average maximum carotid intimamedia thickness. The PASS intervention did not result in clinically important effects, and no other substantial changes were observed. Relatively high probability of clinically beneficial effects of the STEX intervention suggests that a larger, definitive randomized trial with longer follow-up is warranted.
\end{abstract}

A substantial body of scientific work promotes the importance of an active lifestyle in the prevention of CV diseases $(12,22)$. This literature has recently extended to the role of physical activity in the amelioration of risk factors for CV disease in children $(16,18,21)$. Such interest has been prompted, partially, because of reports of increasing levels of childhood obesity and physical inactivity $(19,24,35)$.

Signs and symptoms of clinical CV disease are rarely evident in children. Early (preclinical) noninvasive indicators of $\mathrm{CV}$ disease such as an elevation of carotid intima media thickness (IMT), an increased left ventricular (LV) mass, or a decrease in diastolic function have been used in recent research with children $(9,14,23,32,34)$, however. Of further relevance to such risk factors is the fact that increased physical activity can have a positive impact upon carotid IMT (39), abnormal LV mass (1),

\footnotetext{
*The current study was defined as an exploratory randomized trial, in accordance with the phased approach to the development and evaluation of interventions to improve health proposed by Campbell et al. (2000).
}

Henaghan, McWhannell, Foweather, Cable, Stratton, and George are with Research Institute for Sport and Exercise Sciences (REACH Group), Liverpool John Moores University, UK. Batterham is with the Centre for Food, Physical Activity, and Obesity Research, University of Teesside, UK. 
and diastolic function $(25,27)$. Because of the rarity of clinical vascular events in the young, carotid IMT might be especially relevant as a primary outcome variable for epidemiological and treatment studies in young population samples (20).

Physical activity interventions to reduce CV disease risk in children have tended to involve traditional structured exercise prescription $(1,25,27,39)$, and less attention has been paid to the integration of physical activity into daily life through less-structured sports-specific exercise programs (13). Various theories and models to understand and change lifestyle behavior have been proposed (3) and some recent interventions based on behavior change theories have been studied in an attempt to decrease sedentary behavior in children (30). The comparison of structured exercise interventions with lifestyle programs upon CV risk factors in children has rarely been attempted.

The purpose of this study was, therefore, to assess the impact of a traditional exercise prescription and a lifestyle intervention program upon early prognostic risk factors for $\mathrm{CV}$ disease in primary school children. Both groups were compared with a control group who received no formal physical activity intervention. The current study was defined as an exploratory randomized trial (Phase II), in accordance with the phased approach to the development and evaluation of interventions to improve health proposed by Campbell et al. (6).

\section{Methods}

\section{Participants}

After local ethical approval, schools within Merseyside were recruited via direct contact. Eight schools were selected based on school size (large), availability of school sports facilities (accessible), current afterschool club provision (limited), and socioeconomic status of the area (deprived). Three schools volunteered to participate. After fully informed agreement from head teachers all year, 6 children age 10-11 years $(n=180)$ were informed about the project and asked to return signed parental consent and medical forms. Exclusion criteria included current use of prescription medication, any personal history of asthma or respiratory problems, heart, or vascular complaints as well as an early family history of sudden death. Sixty-one children (36 female and 25 male) 10-11 (11.0 0.3 ) years of age met these criteria, provided written and parental consent, and went on to complete the study.

\section{Design}

Schools were randomly assigned to one of three groups to mitigate potential contamination effects across arms of the trial. The three groups were a structured high-intensity exercise program (STEX), a lifestyle intervention group in a physical activity signposting scheme (PASS); or a control (CON) group. All laboratory data were collected at baseline and after 9 weeks (post intervention).

\section{Physical Activity Signposting Scheme (PASS)}

Social cognitive theory (2) and ecological theory (29) were the basis for the PASS intervention. Nine weekly missions or tasks were sent by post to each child. 
Missions were derived using an intervention mapping approach to try to link the theories specifically to each task with the aim to increase habitual physical activity and decrease sedentary behavior (e.g., to promote active transport and reduce TV viewing). In accordance with the social cognitive theory, children were informed of the benefits of being active including the enhancement of confidence and skill level. Different environmental contexts were used including the home and local neighborhood. In line with the ecological theory, this aimed to help them understand their surroundings and how to become active in it on their own, as well as with family and friends. Pedometers were used as a physical activity promotion tool in conjunction with the missions. Examples of some of the tasks included a "switch off TV challenge" after $2 \mathrm{hr}$ of viewing a day, increasing activity by monitoring active transport, for example, getting off the bus a stop earlier, and taking part in a new activity at least once a week with your family. Ninety-two percent of the children returned the mission in week one and all met the compliance criterion of a $75 \%$ return rate for the duration of the study.

\section{Structured High-Intensity Exercise Program (STEX)}

Children attended twice-weekly hour-long afterschool exercise sessions for 9 weeks. These consisted of multiactivities such as circuits, dance, and games. The aim of the session was to perform whole body muscular activity which was nonsports-specific and to maintain heart rate above $70 \%$ of maximum heart rate $\left(145\right.$ beats $\mathrm{min}^{-1}$ ). This was verified by intermittent heart rate monitoring (Polar Electro, Kempele, Finland). All children met the criteria for attendance of at least $75 \%$ of STEX sessions.

\section{Control (CON)}

The CON group was asked to maintain their normal activity levels. They received the same prestudy information packs as the other two groups, however no further physical activity information was given.

\section{Ultrasound Measurements}

A single trained ultrasound technician performed all ultrasound measurements. Two-dimensional, M-mode, Doppler, and tissue Doppler imaging (TDI) echocardiographic scans were performed using a standard ultrasound system (Mylab30CV system, ESAOTE, Italy) with a $2.5 \mathrm{MHz}$ phased array transducer. All participants where assessed in the left lateral decubitus position.

\section{Carotid Intima-Media Thickness (IMT)}

Ten millimeter segments of the far wall of the common carotid artery (CCA) 1-2 $\mathrm{cm}$ proximal to the carotid bulb were imaged with a $10-15 \mathrm{MHz}$ linear transducer. Four images were taken bilaterally and analyzed off-line (IMT.LABORATORY version 1.1, Pie Medical Equipment, The Netherlands). This software provides average mean and maximum values for carotid IMT via tracking the interfaces of the lumen-intima and media adventitia. 


\section{Cardiac Structure}

Left ventricular structures and LV mass were assessed using parasternal long axis images and M-mode scans at the level of the mitral valve. Wall thickness and cavity dimensions were measured according to the American Society of Echocardiography (31). Left ventricular mass was calculated according to a validated, regressioncorrected formula (11). To determine a size-independent LV mass index, LV mass was divided by height ${ }^{2.7} \cdot(10)$.

\section{Diastolic Function}

From the apical four-chamber view Doppler recordings were taken of mitral inflow by placing a $2 \mathrm{~mm}$ sample volume at the tips of the mitral leaflets and parallel with flow. Peak early (E) and late/atrial (A) flow velocities were obtained and E/A ratio reported. In the same view pulsed TDI velocities were obtained from the septal annular site using a $2 \mathrm{~mm}$ sample volume. Peak early diastolic (E') and late diastolic (A') myocardial tissue velocities were recorded and $\mathrm{E}^{\prime} / \mathrm{A}^{\prime}$ ratio was derived. The intraobserver coefficient of variance for $\mathrm{LVM}, \mathrm{E}^{\prime} / \mathrm{A}^{\prime}$ ratio and cIMT was reported as $7.4 \%, 11.5 \%$ and $1.2 \%$ respectively.

\section{Other Outcome Measures}

Standard anthropometrics including body mass (Seca Limited, Birmingham UK), stature (Leister height measure, Birmingham, UK), and body mass index (weight $[\mathrm{kg}] /$ height $[\mathrm{m}]^{2}$ ) were recorded. Body composition was assessed using dual-energy X-ray absorptiometry (DEXA; Lunar Hologic QDR, USA) in which the whole body was scanned. Key variables assessed were lean body mass $(\mathrm{kg})$, fat mass $(\mathrm{kg})$, and percent body fat. After 5 min of supine-seated rest, brachial artery blood pressures and heart rate (Bosch and Sohn, Germany) were assessed twice with the lowest score noted.

\section{Statistical Analysis}

Values are presented as mean $\pm S D$. The carotid IMT was defined a priori as the primary outcome variable, with the other measured variables classed as secondary outcomes. Analysis of covariance was conducted to evaluate the effectiveness of the 9-week interventions. The independent variable was the type of intervention (STEX or PASS vs. CON), with the dependent variable as the change score (post minus baseline). The baseline score served as the covariate to control for chance imbalances across groups at baseline (38). Exploratory analyses indicated no substantial interactions with gender; therefore, boys and girls were merged into a single group for the primary analysis. An estimation approach was considered superior to a null hypothesis testing framework (8), presenting the mean effects of each intervention (versus control) on the primary outcome, together with $90 \%$ confidence intervals as suggested by Sterne and Smith (37). In line with the recommendations of Perneger (28), Bonferroni corrections of confidence intervals were not applied.

Adjusted mean intervention effects were evaluated for their clinical significance by prespecifying the minimum clinically importance difference (MCID; 4). In the absence of a robust clinical anchor, the MCID is defined conventionally 
using a distribution-based method as a Cohen's $d$ (difference in the change scores between groups) of 0.2 between subject standard deviations (7). The SD of the pooled baseline scores was used for this purpose, because the posttest SD might be inflated by individual differences in responses to the intervention. The MCID was interpreted as "benefit" or "harm" according to the direction of the effect on the intervention for a given variable. As a Phase II exploratory trial, the current study is not powered to precisely define changes as small as the MCID. The effects observed, however, will provide important information to inform sample-size estimations in any subsequent Phase III trial (6).

Using the mean intervention effect together with its uncertainty, the probability (percent chances) that the true population effect was as least as large as the MCID was calculated $(4,15,33)$. Briefly, this process requires the calculation of a $t$ statistic for the intervention effect. The conventional $t$ statistic involves a test against the null hypothesis of zero effect ( $t=$ (mean difference between groups-zero)/ standard error of the difference). To calculate the probability of clinical benefit, the zero in this formula is replaced by the prespecified value for the MCID: $t=$ (mean difference between groups-MCID)/ standard error of the difference. With the correct degrees of freedom for the comparison, the area under the $t$ distribution curve to the left of the $|t|$ value returns the probability that the true population effect of the intervention is at least as large as the MCID. All analyses were carried out on SPSS statistical package 14.0 (SPSS Inc., Chicago, USA).

\section{Results}

Data for age, body size and composition is presented in Table 1. Between-group differences in variables at baseline were not substantial although there was a trend for a greater BMI, waist circumference, fat mass and percent body fat in the control group. After the 9-week intervention there were no clinically substantial differences in $\Delta$ scores between groups for all body size variables.

Data from cardiovascular primary and secondary outcomes are presented in Table 2 . There were no clinically substantial intervention effects for any secondary variable. Figure 1 and 2 demonstrate the mean $\Delta$ scores (adjusted for baseline imbalance) for average mean and average maximum cIMT, respectively. The minimum clinically important difference (MCID), defined as 0.2 between-subject standard deviations, was $0.008 \mathrm{~mm}$ for both average mean cIMT and average maximum cIMT. The effect of the STEX intervention (compared with CON) was a mean benefit of $-0.018 \mathrm{~mm}$ for average maximum cIMT (90\% CI, $-0.039-0.002$ $\mathrm{mm}$ ), and $-0.016 \mathrm{~mm}$ for average mean cIMT (90\% CI, $-0.040-0.008 \mathrm{~mm}$ ). The probability (\% chances) that the effect of the STEX intervention was at least the MCID was $79 \%$ for average maximum and $71 \%$ for average mean carotid IMT. The PASS intervention did not result in clinically important effects.

\section{Discussion}

To the authors' knowledge, this is the first exploratory trial to assess the impact of both a structured exercise program, as well as a lifestyle intervention upon early risk factors for $\mathrm{CV}$ disease in children. Whereas most body composition and $\mathrm{CV}$ 
Table 1 Anthropometrics Data in All Groups Pre- and Postintervention

\begin{tabular}{|c|c|c|c|c|c|c|}
\hline & CON & $=30)$ & PASS & $=15)$ & STEX & $=16)$ \\
\hline & Mean & & Mean & & Mean & \\
\hline & Value & $S D$ & Value & $S D$ & Value & $S D$ \\
\hline Age (yr) & & & & & & \\
\hline Baseline & 11.0 & $(0.3)$ & 11.0 & $(0.3)$ & 11.1 & $(0.3)$ \\
\hline Posttest & 11.2 & $(0.3)$ & 11.2 & $(0.4)$ & 11.3 & $(0.3)$ \\
\hline$\Delta$ & 0.2 & $(0.3)$ & 0.2 & $(0.3)$ & 0.2 & $(0.3)$ \\
\hline Body mass (kg) & & & & & & \\
\hline Baseline & 40.7 & (7.6) & 40.7 & (8.9) & 41.4 & $(10.7)$ \\
\hline Posttest & 44.8 & (9.4) & 42.4 & (9.6) & 42.9 & (11.1) \\
\hline$\Delta$ & 4.1 & (7.4) & 1.7 & (8.4) & 1.5 & (10.6) \\
\hline Stature (m) & & & & & & \\
\hline Baseline & 1.48 & $(0.09)$ & 1.44 & $(0.07)$ & 1.48 & $(0.07)$ \\
\hline Posttest & 1.49 & $(0.09)$ & 1.46 & $(0.07)$ & 1.50 & $(0.07)$ \\
\hline$\Delta$ & 0.01 & $(0.09)$ & 0.02 & $(0.07)$ & 0.02 & $(0.07)$ \\
\hline $\operatorname{BMI}\left(\mathrm{kg} / \mathrm{m}^{2}\right)$ & & & & & & \\
\hline Baseline & 19.8 & (3.1) & 19.3 & $(3.0)$ & 18.7 & (3.3) \\
\hline Posttest & 20.0 & (3.1) & 19.7 & (3.3) & 18.8 & (3.3) \\
\hline$\Delta$ & 0.2 & $(0.5)$ & 0.4 & $(0.5)$ & 0.1 & (0.3) \\
\hline $\begin{array}{l}\text { Waist circumfe } \\
(\mathrm{cm})\end{array}$ & & & & & & \\
\hline Baseline & 69.6 & (9.6) & 68.0 & $(10.0)$ & 65.6 & (10.9) \\
\hline Posttest & 70.9 & $(10.8)$ & 68.5 & $(9.5)$ & 64.9 & (10.9) \\
\hline$\Delta$ & 1.3 & $(4.7)$ & 0.5 & $(2.7)$ & -0.7 & $(-2.7)$ \\
\hline Fat mass $(\mathrm{g})$ & & & & & & \\
\hline Baseline & 12018 & (5240) & 10618 & (4547) & 11452 & (6324) \\
\hline Posttest & 12667 & (5431) & 11394 & (4831) & 11819 & (6687) \\
\hline$\Delta$ & 649 & (966) & 776 & (904) & 368 & (563) \\
\hline Lean mass (g) & & & & & & \\
\hline Baseline & 30383 & (5047) & 29234 & (5168) & 29060 & (4886) \\
\hline Posttest & 31265 & (5136) & 30226 & (5628) & 30307 & (4995) \\
\hline$\Delta$ & 882 & $(845)$ & 992 & $(826)$ & 1246 & (796) \\
\hline$\%$ Fat mass $(\%$ & & & & & & \\
\hline Baseline & 26.7 & (7.4) & 25.1 & (5.8) & 26.2 & (7.3) \\
\hline Posttest & 27.1 & (7.1) & 25.7 & (5.9) & 26.0 & (7.6) \\
\hline$\Delta$ & 0.4 & (7.3) & 0.6 & $(5.8)$ & -0.2 & (7.6) \\
\hline
\end{tabular}

risk factor variables did not change meaningfully, an interesting and potentially clinically relevant change in cIMT was observed in the STEX group compared with CON. On this basis, cIMT might be a more sensitive variable than traditional risk factors and other variables assessed within the current study to track changes in $\mathrm{CV}$ risk due to physical activity interventions (5). 
Table 2 Cardiovascular Data in All Groups Pre- and Postintervention

\begin{tabular}{|c|c|c|c|c|c|c|}
\hline & \multicolumn{2}{|c|}{$\begin{array}{l}\text { Control group } \\
(n=30)\end{array}$} & \multicolumn{2}{|c|}{$\begin{array}{c}\text { PASS } \\
(n=15)\end{array}$} & \multicolumn{2}{|c|}{$\begin{array}{c}\text { STEX } \\
(n=16)\end{array}$} \\
\hline & Mean & & Mean & & Mean & \\
\hline & Value & $S D$ & Value & $S D$ & Value & $S D$ \\
\hline \multicolumn{7}{|c|}{ Systolic BP (mmHg) } \\
\hline Baseline & 126 & (26) & 130 & (34) & 113 & (24) \\
\hline Posttest & 134 & (30) & 137 & (41) & 123 & (39) \\
\hline$\Delta$ & 8 & (21) & 7 & (23) & 10 & (23) \\
\hline \multicolumn{7}{|c|}{ Diastolic BP (mmHg) } \\
\hline Baseline & 67 & (7) & 68 & (8) & 68 & (4) \\
\hline Posttest & 62 & (5) & 61 & (7) & 62 & (5) \\
\hline$\Delta$ & -5 & (6) & -7 & (8) & -6 & (6) \\
\hline \multicolumn{7}{|c|}{$\begin{array}{l}\text { Resting heart rate } \\
\text { (bpm) }\end{array}$} \\
\hline Baseline & 73 & (10) & 75 & (9) & 74 & (11) \\
\hline Posttest & 70 & (9) & 73 & (10) & 71 & (8) \\
\hline$\Delta$ & -3 & (9) & -2 & (9) & -3 & (9) \\
\hline \multicolumn{7}{|l|}{ LV mass (g) } \\
\hline Baseline & 126 & (26) & 130 & (34) & 113 & (24) \\
\hline Posttest & 134 & (30) & 137 & (41) & 123 & (39) \\
\hline$\Delta$ & 8 & (21) & 7 & (23) & 10 & (23) \\
\hline \multicolumn{7}{|c|}{$\begin{array}{l}\text { LV mass indexed (g/ } \\
\mathrm{m} 2 \text { ) }\end{array}$} \\
\hline Baseline & 42.1 & $(10.5)$ & 47.6 & (9.6) & 39.8 & $(6.8)$ \\
\hline Posttest & 45.0 & $(7.0)$ & 48.8 & (12.4) & 40.2 & (7.9) \\
\hline$\Delta$ & 2.8 & (10.7) & 1.2 & $(8.1)$ & 2.4 & $(7.9)$ \\
\hline \multicolumn{7}{|l|}{$\mathrm{E} / \mathrm{A}$ ratio $(\mathrm{m} / \mathrm{s})$} \\
\hline Baseline & 2.12 & $(0.52)$ & 1.98 & $(0.45)$ & 2.04 & $(0.47)$ \\
\hline Posttest & 2.05 & $(0.50)$ & 1.87 & $(0.41)$ & 2.01 & $(0.55)$ \\
\hline$\Delta$ & -0.07 & $(0.42)$ & -0.11 & $(0.31)$ & -0.03 & $(0.51)$ \\
\hline \multicolumn{7}{|c|}{$\mathrm{E}^{\prime} / \mathrm{A}^{\prime}$ ratio $(\mathrm{cm} / \mathrm{s})$} \\
\hline Baseline & 2.65 & $(0.70)$ & 2.24 & $(0.57)$ & 2.58 & $(0.67)$ \\
\hline Posttest & 2.85 & $(0.60)$ & 2.36 & $(0.50)$ & 2.62 & $(0.67)$ \\
\hline$\Delta$ & 0.20 & $(0.64)$ & 0.12 & $(0.53)$ & 0.04 & $(0.51)$ \\
\hline \multicolumn{7}{|c|}{$\begin{array}{l}\text { Average mean cIMT } \\
(\mathrm{mm})\end{array}$} \\
\hline Baseline & 0.389 & $(0.043)$ & 0.409 & $(0.032)$ & 0.423 & $(0.039)$ \\
\hline Posttest & 0.400 & $(0.045)$ & 0.405 & $(0.067)$ & 0.402 & $(0.029)$ \\
\hline$\Delta$ & 0.011 & $(0.039)$ & -0.004 & $(0.061)$ & -0.021 & $(0.047)$ \\
\hline \multicolumn{7}{|c|}{$\begin{array}{l}\text { Average max cIMT } \\
(\mathrm{mm})\end{array}$} \\
\hline Baseline & 0.405 & $(0.041)$ & 0.425 & $(0.031)$ & 0.440 & $(0.040)$ \\
\hline Posttest & 0.419 & $(0.046)$ & 0.437 & $(0.053)$ & 0.423 & $(0.026)$ \\
\hline$\Delta$ & 0.014 & $(0.035)$ & 0.011 & $(0.045)$ & -0.017 & $(0.043)$ \\
\hline
\end{tabular}




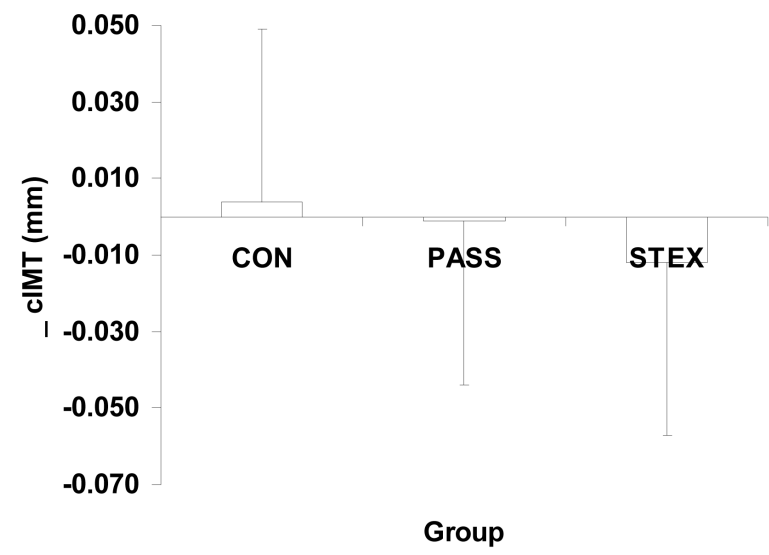

Figure $1-\Delta$ scores for adjusted average mean carotid IMT over the 9-week intervention period in all groups.

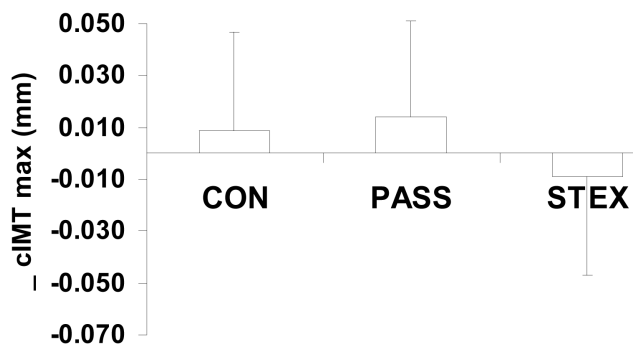

\section{Group}

Figure $2-\Delta$ scores for adjusted average maximum carotid IMT over the 9-week intervention period in all groups.

The current data partially supports previous research $(36,39)$ that reported decreased cIMT with exercise training and extends this data to suggest that even short-term interventions may produce favorable responses in cIMT. Research with adults has proposed that an increase in cIMT of just $0.034 \mathrm{~mm}$ a year is an independent risk factor for CV disease (17). Similarly, a 1 SD increase in cIMT was associated with a twofold increased risk of ischemic stroke or myocardial infarction (26). Such data are unavailable for the age range in the current study, so the MCID was defined as 0.2 between-subject standard deviations - a reduction in cIMT of $0.008 \mathrm{~mm}$. The calculated probability that the effect of the STEX intervention exceeded this threshold (defining clinical benefit for reduction in cIMT) was relatively high (0.71-0.79). For average maximum cIMT, the calculated probability reflected odds of approximately 4:1 in favor of the STEX intervention being clinically beneficial, with odds of greater than $2: 1$ that the intervention was beneficial for average mean cIMT. 
Clearly, other researchers and clinicians might disagree with the value specified in the current study for the MCID. For those desiring a different threshold to define the MCID, an example of a clinical significance curve, as recommended by Shakespeare et al. (33), is presented in Figure 3. In the current study, prespecifying a larger MCID of half a standard deviation $(0.02 \mathrm{~mm})$ for average maximum cIMT, for example, results in a probability of clinical benefit of 0.44 (indicating that the intervention would be about as likely to be clinically worthwhile as not). Half a standard deviation, however, represents a moderate effect size, and it is believed that even small changes of carotid IMT might be clinically relevant at a population level (39). Certainly, the findings provide sufficiently robust evidence of STEX benefit to warrant further investigation in a definitive randomized controlled trial. The PASS group did not produce such positive results for average mean and maximum cIMT. This is likely to be a result of a reduced intensity, duration and thus volume of physical activity in PASS compared with STEX.

Changes in other CV risk variables were not substantially different between groups. This likely reflects the short nature of the intervention or the relative health of the heterogeneous primary school population. A longer intervention may be warranted to assess the impact of STEX and PASS on LV mass and diastolic function possibly in more "at risk" children. The lack of change in diastolic functional variables is somewhat at odds with past research $(25,27)$ but is likely because these data were derived from cross-sectional comparisons of trained and untrained children, which represents a greater exercise stimulus accumulated over many years. Moreover, it is almost axiomatic that cross-sectional effects are larger than those observed in experimental designs.

As with CV risk factors, there were no substantial between group differences in $\Delta$ scores for body size and composition variables. Of some interest, however, was a small decrease in waist circumference and percentage fat mass in the STEX group compared with a small increase in the CON and PASS groups. Such group differences would be interesting to explore further in a more prolonged intervention.

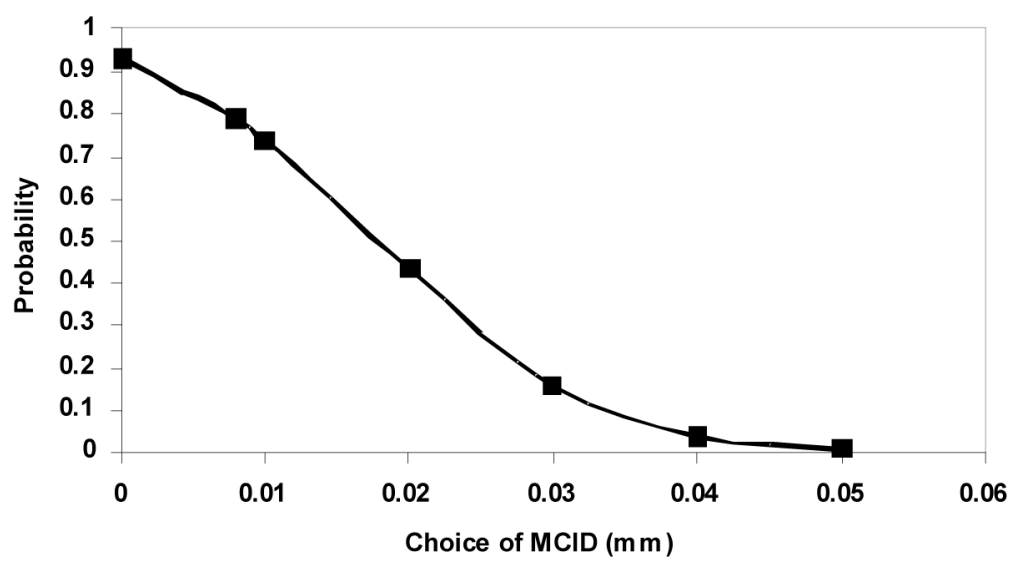

Figure 3 - The probability that the true population intervention effect exceeds a chosen Minimum Clinically Important Difference (MCID; change in average maximum carotid IMT). 
Of potential interest to future research was the relative success of the implementation of both the PASS and STEX interventions in primary schoolchildren. As an exploratory trial, it was positive to observe participants attain a $75 \%$ criterion for attendance or completion of signposting tasks. In addition, a poststudy review of attendance and return, logistics of delivery, and logistics of testing and assessment were all positive. Positive feedback was also given from questionnaires completed by participants and parents. It is, though, pertinent to note some lessons for future studies. In the PASS scheme, response rates varied task to task and generally declined over the 9 -week period. It is recommended that attention needs to be paid to nature of the tasks including an attempt to enhance the intensity of the program similar to that of the STEX group. Implementation of positive reinforcement such as a reward scheme could be introduced, as well as structured support from the child's school and family is also important to help maintain adherence. In addition, data related to habitual physical activity levels would be useful and provide extra insight into the effectiveness of the specific interventions. Physical activity data collection was attempted in this exploratory trial, but logistical and technical problems prevented these data being included in the analyses.

On the basis of the current findings and the lessons learned from this exploratory study, we propose the implementation of a reward scheme to reinforce positive behavior change in addition to a longer intervention period with a larger sample size in a definitive (Phase III) randomized controlled trial (6). A larger trial would enable the potential clinical benefit of the intervention to be estimated more precisely. The targeting of children with higher risk of cardiovascular disease would also be valuable (e.g., overweight and obese). In addition, in future research the impact that the STEX and PASS interventions have on habitual physical activity and associated energy expenditure should be considered.

In conclusion, this exploratory trial of a STEX and PASS intervention in primary schoolchildren showed the interventions to be feasible, whereas the STEX intervention produced small but potentially clinically beneficial changes in the cIMT primary outcome variable. The focus of the current study on quantifying the probability for the potential benefit of the intervention encourages researchers and clinicians to justify their choice of the smallest clinically worthwhile effect and enhances clinical decision-making (33). Future research might provide greater insight into changes in cIMT after exercise interventions in children but might wish to target longer training periods and more at risk cohorts.

\section{References}

1. Bacon, S.L., A. Sherwood, A. Hinderliter, and J.A. Blumenthal. Effects of exercise, diet and weight loss on high blood pressure. Sports Med.34:307-316, 2004.

2. Bandura, A. Social Foundations of Thought and Action: A Social Cognitive Theory. Englewood Cliffs, NJ: Prentice Hall, 1986.

3. Baranowski, T., K.W. Cullen, T. Nicklas, D. Thompson, and J. Baranowski. Are current health behavioural change models helpful in guiding prevention of weight gain effort? Obes. Res. 11:23S-43S, 2003.

4. Batterham, A.M., and W.G. Hopkins. Making meaningful inferences about magnitudes. Int J Sport Phys Perf. 1:50-57, 2006.

5. Bots, M.L. Carotid intima-media thickness as a surrogate marker for cardiovascular disease in intervention studies. Curr. Med. Res. Opin. 22:2182-2190, 2006. 
6. Campbell, M., R. Fitzpatrick, A. Haines, et al. Framework for design and evaluation of complex interventions to improve health. BMJ. 321:694-696, 2000.

7. Cohen, J. Statistical Power Analysis for the Behavioral Sciences (2nd ed.). Hillsdale, NJ: Lawrence Erlbaum, 1988.

8. Curran-Everett, D., S. Taylor, and K. Kafadar. Fundamental concepts in statistics: elucidation and illustration. J. Appl. Physiol. 85:775-786, 1998.

9. Daniels, S.R., J.A. Morrison, D.L. Sprecher, P. Khoury, and T.R. Kimball. Association of body fat distribution and cardiovascular risk factors in children and adolescents. Circulation. 2:541-545, 1999.

10. De Simone, G., J.R. Kizer, M. Chinali, et al. Normalization for body size and population-attributable risk of left ventricular hypertrophy: the Strong Heart Study. Am. J. Hypertens. 18:191-196, 2005.

11. Devereux, R.B., D.R. Alonso, E.M. Lutas, et al. Echocardiographic assessment of left ventricular hypertrophy: comparison of necropsy findings. Am. J. Cardiol.57:450-458, 1986.

12. Farrell, S.W., J.B. Kampert, H.W. Kohl, et al. Gibbon, and S.N. Blair. Influences of cardiorespiratory fitness levels and other predictors on cardiovascular disease mortality in men. Med. Sci. Sports Exerc. 30:899-905, 1998.

13. Floriani, V., and C. Kennedy. Promotion of physical activity in primary car for obesity treatment/prevention in children. Curr. Opin. Pediatr. 19:99-103, 2007.

14. Freedman, D.S., W.H. Dietz, R. Tang, et al. The relation of obesity throughout life to carotid intima-media thickness in adulthood: the Bogalusa Heart Study. Int. J. Obes. 28:159-166, 2004.

15. Froehlich, G. What is the chance that this study is clinically significant? A proposal for Q values. Eff. Clin. Pract. 2:234-239, 1999.

16. Harrell, J.S., P.F. Pearce, and L.L. Hayman. Fostering prevention in paediatric population. J. Cardiovasc. Nurs. 18:144-149, 2003.

17. Hodis, H.N., W.J. Mack, L. Labree, R.H. Selzer, C. Liu, and P.A. Stanley. The role of carotid intima-media thickness in predicting clinical coronary events. Ann. Intern. Med. 128:262-269, 1998.

18. Invitti, C., C. Maffeis, L. Gilardini, et al. Metabolic syndrome in obese Caucasian children: prevalence using WHO-derived criteria and association with non-traditional cardiovascular risk factors. Int. J. Obes. 30:627-633, 2006.

19. Lobstein, T., and M.L. Frulet. Prevalence of overweight among children in Europe. Obes. Rev. 4:195-200, 2003.

20. Lorenz, M.W.,H.S. Markus, M.L. Bots, M. Rosvall, and M. Sitzer. Prediction of clinical cardiovascular events with carotid intima-media thickness. A systematic review and meta-analysis. Circulation. 115:459-467, 2007.

21. McGill, H.C., A. McMahan, E.E. Hererick, et al. Effects of coronary heart disease risk factors on atherosclerosis of selected regions of the aorta and right coronary artery. Arterioscler. Thromb. Vasc. Biol. 20:836-845, 2000.

22. Mertens, D.J., T. Kavanagh, R.B. Campbell, and R.J. Shepard. Exercise without dietary restriction as a means to long term fat loss in the obese cardiac patient. J Sports Med Phys Fitness. 38:310-316, 1998.

23. Meyer, A.A., G. Kundt, M. Steiner, P. Schuff-Werner, and W. Kienast. Impaired flowmediated vasodilation, carotid artery imtima-media thickening, and elevated endothelial plasma markers in obese children: the impact of cardiovascular risk factors. Pediatrics. 177:1560-1567, 2006.

24. Nelson, M.C., D. Neumark-Stzainer, P.J. Hannan, J.R. Sirard, and M. Story. Longitudinal and secular trends in physical activity and sedentary behaviour during adolescence. Pediatrics. 118:e1627-e1634, 2006.

25. Nottin, S., L-D. Nguyen, M. Terbah, and P. Obert. Left ventricular function in endurance trained children by tissue Doppler imaging. Med. Sci. Sports Exerc. 36:1507-1513, 2004. 
26. O’Leary, D.H., J.F. Polak, R.A. Kronmal, T.A. Manolio, G.L. Burke, and S.K. Jr Wolfson. Carotid-artery intima and media thickness as a risk factor for myocardial infarction and stroke in older adults. N. Engl.J. Med. 340:14-22, 1999.

27. Pela, G., G. Bruschi, L. Montagna, M. Manara, and C. Manca. Left and right ventricular adaptation assessed by Doppler tissue echocardiography in athletes. J. Am. Soc . Echocardiogr. 17:205-211, 2004.

28. Perneger, T.V. What's wrong with Bonferroni adjustments? BMJ. 316:1236-1238, 1998.

29. Sallis, J.F., and N. Owen. Ecological models. In: Health behaviour and health education: theory, research, and practice (2nd ed.). K. Glanz, F.M. Lewis, and B.K. Rimmer (Eds.). San Francisco: Jossey-Bass, 1999.

30. Salmon, J., K. Ball, D. Crawford, et al. Reducing sedentary behaviour and increasing physical activity among 10-year-old children: overview and process evaluation of the 'switch-play' intervention. Health Promot Int. 20:7-17, 2005.

31. Schiller, N.B., P.M. Shah, M. Crawford, et al. Recommendations for quantitation of the left ventricle by two-dimensional echocardiography. American Society of Echocardiography Committee on Standards, Subcommittee on Quantitation of two-dimensional echocardiograms. J. Am. Soc. Echocardiogr. 2:358-367, 1989.

32. Sharpe, J.A., L.H. Naylor, T.W. Jones, et al. Impact of Obesity on diastolic function in subjects $\leq 16$ years of age. Am. J. Cardiol. 98:691-693, 2006.

33. Shakespeare, T.P., V.J. Gebski, M.J. Veness, and J. Simes. Improving interpretation of clinical studies by use of confidence levels, clinical significance curves, and risk-benefit contours. Lancet. 357:1349-1353, 2001.

34. Sorof, J.M., A.V. Alexandrov, Z. Garami, et al. Carotid ultrasonography for diction of vascular abnormalities in hypertensive children. Pediatr. Nephrol. 18:1020-1024, 2003.

35. Sproston, K., and P. Primatesta. Health Survey for England 2002: The Health of Children and Young People. London: The Stationery Office, 2003.

36. Stein, J.H., P.S. Douglas, S.R. Srinivasan, et al. Distribution and cross-sectional agerelated increases of carotid artery intima-media thickness in young adults. The Bogalusa Heart Study. Stroke. 35:2782-2787, 2004.

37. Sterne, J.A., and G. Davey Smith. Sifting the evidence - what's wrong with significance tests? BMJ. 322:226-231, 2001.

38. Vickers, A.J., and D.G. Altman. Statistics Notes: Analysing controlled trials with baseline and follow up measurements. BMJ. 232:1123-1124, 2001.

39. Woo, K.S., P. Chook, C.W. Yu, et al. Effects of diet and exercise on obesity related vascular dysfunction in children. Circulation. 109:1981-1986, 2004. 